\title{
Context for costumes: faceted access to historic costumes
}

\author{
Arden Kirkland \\ Vassar College \\ Poughkeepsie, NY USA \\ arkirkland@vassar.edu
}

\author{
Michael Lesk \\ Rutgers University \\ New Brunswick, NJ USA \\ lesk@acm.org
}

\author{
Allison Steffmann \\ Rutgers University \\ New Brunswick, NJ USA \\ allison@steffmann.com
}

\begin{abstract}
Historic costumes, like many other kinds of museum objects, benefit from remote access to imagery: they are fragile, widely scattered, and of interest to scholars who may not find it easy to visit every possible repository. Cataloguing them involves multiple facets. Researchers may be interested in organisation by purpose, material, period, designer, or construction, whereas original descriptions may focus on colour or size. Vocabulary may be either current ('blouse') or obsolete ('shirtwaist'). And, as usual, some users will be posing carefully phrased queries while others are in a browsing mode. In common with many museum areas, images are also essential for many users. We are also experimenting with context; many costume collections are relatively small, and provision of an expanded view of an area may assist users.
\end{abstract}

Cataloguing. Costumes. VR. Facets. Vocabulary

\section{METADATA: AN IDEA WHOSE TIME HAS PASSED?}

Should items in a catalogue be organised in some subject-oriented method, or accessed solely by keyword search? Until the advent of the Web, it was normally assumed that library contents would be organised in some way, whether in a large formal structure such as the Dewey Decimal classification, or in some less formal system of folders and headings. Search engines have made it commonplace for users to just assume that keyword search will be a substitute. In museum contexts a great many different kinds of organisations may be possible, and in addition the presence of novice users (often more common in museums than in archives) makes it desirable to provide browsing. Experiments have long distinguished between different kinds of users: those who are familiar with the contents of a collection may be better off searching, while those who are not may be better off with a method for browsing (Geller \& Lesk, 1983).

The costs of metadata creation are making it more and more difficult for any formal structure to be adopted. It is not helpful that standards groups create ever more elaborate metadata systems which make the creation of metadata even more expensive, although the use of Dublin Core and its derivatives does something to simplify matters. In the costume area, the Visual Resources Association has compiled the VRA Core as an extension to the basic Dublin Core structure (VRA, 2010). As a comparison, the web page describing VRA Core is about 10,000 bytes; the Content Standard for Digital Geospatial Metadata, 90 pages, is 164,000 bytes.

Whether metadata as such is highly valuable has become a matter of dispute. The Library of Congress prepared a report questioning the value of traditional cataloguing (Calhoun, 2006), and the MIT Libraries feel it necessary to provide a publication entitled The Value of Metadata in the Google Era (Wolfe, 2010). Other writers, however, argue for the continued importance of metadata to provide and distinguish indicators of the content, the context, and the inter-relationships of objects (Gilliland-Swetland, 1998).

Metadata for costume collections is sometimes challenging when only a few items are held in one institution as part of a much larger general collection. In that circumstance it is probable that the overall cataloguing strategy will be driven by the needs of the collection as a whole, with only minor attention given to any special terminology for costumes. For example, Fry and Riley complain about items being indexed only in general terms

*To whom correspondence should be addressed. 
even though they are relevant to very specific costume queries (Fry \& Riley, 2006).

\section{ORGANISATION FROM TEXT}

One possible way of replacing or supplementing conventional metadata would be user tagging or 'folksonomies'. However, costume material is extremely specialised, and there is a fear than amateurs will not know the right categories or words (see Fry \& Riley, 2006). Perhaps a reasonable compromise is to begin with a written description of an item, written by a specialist who does know the right words to describe something, but is perhaps not an expert in metadata formats and styles. Using such descriptions, can we supplement traditional metadata with automatically organised material, using some kind of text mining or classification?

In particular, different kinds of organisation might be reflected in different arrangements of items. For example, compare the Getty Art \& Architecture thesaurus, which is arranged functionally, with the Encyclopaedia Britannica 11th edition article on 'Costume', which is arranged historically. Figure 1 below compares the distribution in these texts of the words shoe, Greek, and linen. In each of the three subfigures the red boxes on the left show appearances in the Getty Art \& Architecture Thesaurus, while the blue boxes on the right show the appearances in the Encyclopaedia Britannica

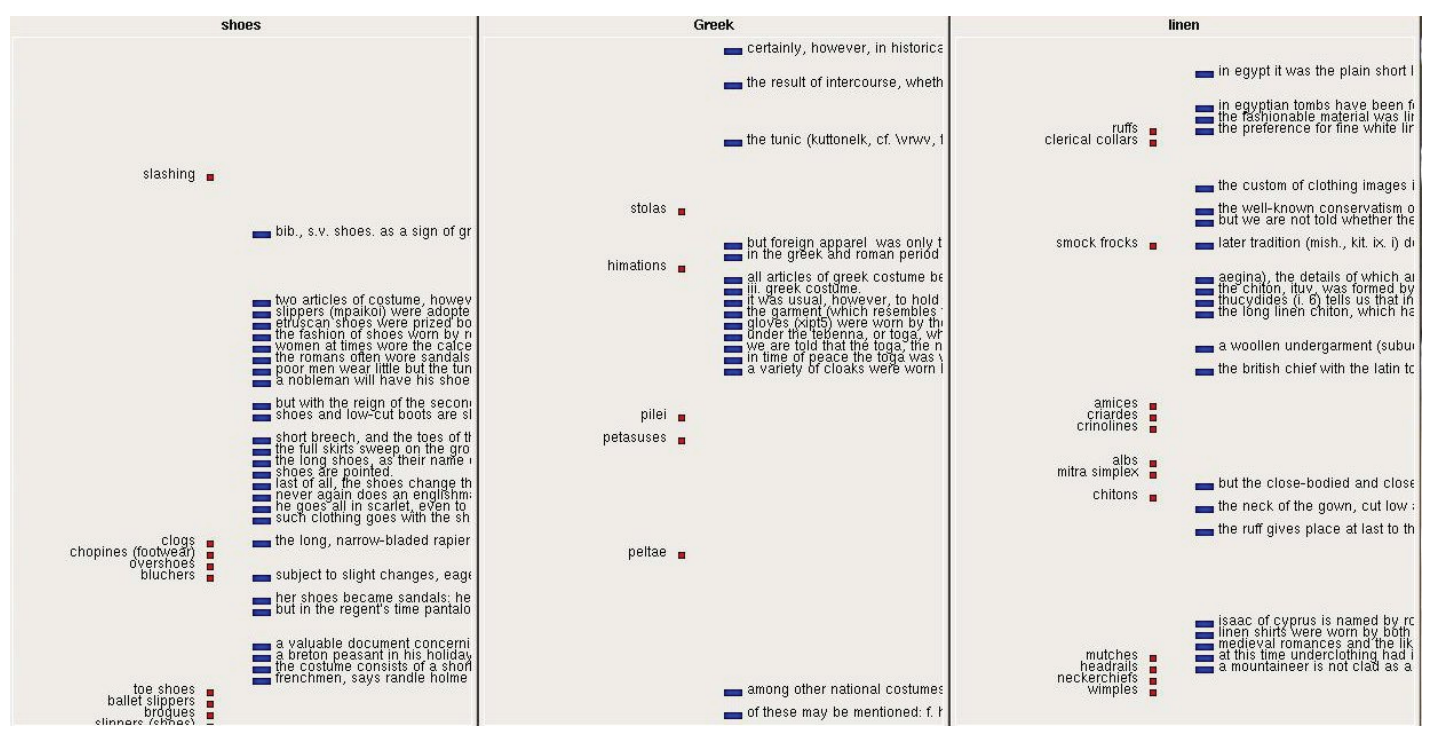

Figure 1: Comparison of word distributions

article. As you can see, the functional term (shoe) is grouped much more tightly in the Getty thesaurus than the encyclopaedia, while the historical term (Greek) is more tightly grouped in the encyclopaedia. The material term (linen) whose idea is central to neither organisation is spread widely in each form.

In the context of costume history, other clearly relevant organisations would be based on materials, techniques, designers, time periods, places, and styles. Sizes and colours are also of value to some researchers although they do not have the general importance that they would have for someone planning to wear the clothes. Ideally, we would like to have a collection of reference taxonomies or essays into which we would fit items automatically, just by term matching between the descriptions of the objects and the text sequence in the references. For this purpose a dictionary is not quite enough, since we need to sequence or at least group items, requiring hierarchical information For example, if Wordnet (Fellbaum, 1998) had an adequate supply of special costume terms, it would have been an additional potential organisation of our material.

Automatic hierarchy creation (Stoica et al., 2007) would be another alternative, rather than relying on the structure of an original reference document. If successful it would permit more sophisticated placement of items than simple linear position within a document, but it requires more information on the input side. Given the number of facets that are relevant for costume it seems desirable to require the simplest possible input, in the hope of being able to accept a larger number and variety of reference documents, than to make greater demands on the classification methods. 


\section{PLACEMENT}

Assuming that one does have a reference document that is believed to discuss related topics together, one can then display items from a particular costume collection in some organisation that reflects that particular reference organisation.

The goal is to let the user browse items that have been grouped into some specific organisation. This would serve a user who is unsure of the correct words to use in searching a description, likely to be a common problem in costume collections as a result of terminology shifts across the decades.

For example, Figure 2 below shows the result of plotting a few items from the Vassar costume collection in (on the left) the sequence implied by the Getty thesaurus and then (on the right) in a sequence implied by the Encyclopaedia Britannica article, which doesn't know the word gymsuit, and mashes everything together as 'dress'.

The intent of this interface is to provide a method of browsing for a novice user. We anticipate that there will be several different levels of use for any costume catalogue, ranging from the scholars who know not only the right words but the facet to which they apply. In the same way that bibliographic interfaces can be searched by title or author or date, a costume collection might be searched by designer name, the description of a garment, or the material of which something is made (Yee et al., 2003) Such a searcher would be able to distinguish between a search for something worn by Lauren Hutton or designed by Ralph Lauren. Other users, however, will not know the correct terms for older materials and may wish to avoid faceted searching, being accustomed to simpler interfaces.

This particular interface works as follows. There is a 'reference document' which is simply a long text describing costumes. It is written in some coherent order so that position in this document somehow reflects properties of the objects described. Then, the text description of each object in turn is searched in this reference document, and the location of the best match (counting word overlaps) is used as a content indicator, with the title of the object and a thumbnail picture placed in the corresponding location.

The major problem, aside from the difficulty of finding a wide selection of good reference documents, is simply screen space: if each object needs a thumbnail, only a few can be displayed. We imagine the user starting with a few words that are used for ordinary text search in the object descriptions, so that what this interface does is to order rather than select items for view.

A proposed extension to deal with a larger number of images would be to use a 'fisheye' style of display (Furnas, 1986). In this style of display, a few items that were judged 'central' would be shown in reasonable detail, with peripheral items being reduced to a few words or dots. As the user adjusted the viewpoint, the smaller items would be increased in visibility. This would permit a large number of items to be 'shown' with detail available on each one as needed.

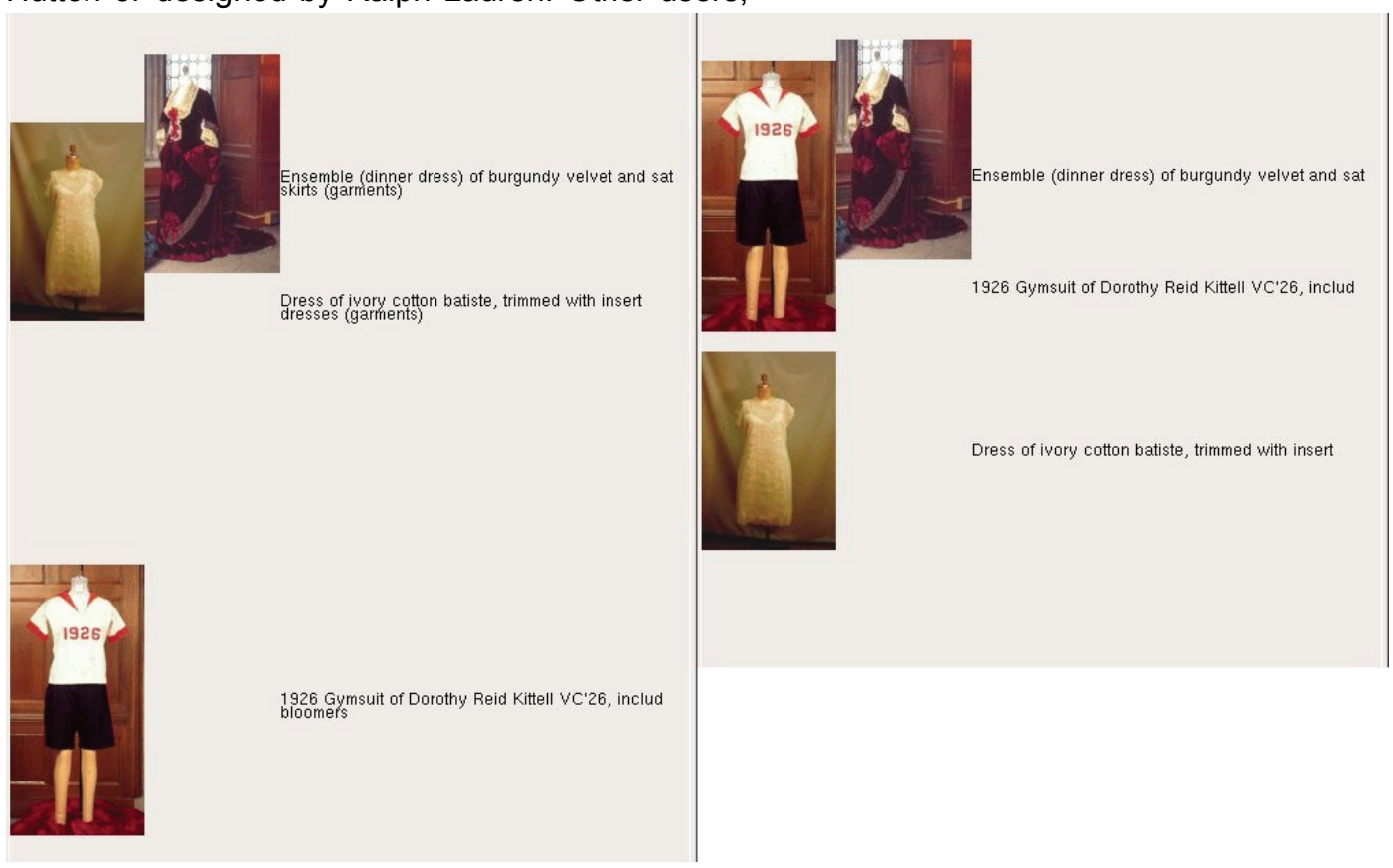

Figure 2: Distribution of images in Getty text $(L)$ or encyclopaedia $(R)$ 


\section{CONTEXT}

Again thinking about the user who is not immediately familiar with the resources available for costume history, the question of seeing the context for any particular garment may be important. Given one image, what are the other images that are closely related to it? This could be phrased in terms of items in the same collection, items in other costume collections, or even more general full web resources. It would be valuable to allow extension of searches beyond a single costume collection, while still maintaining some kind of base display so that the user run a risk of becoming 'lost in hyperspace.' Depending on the circumstances, both local and more distant context might be relevant.

We thus looked at the problem of how to find related items in a wider context. This might involve other items in the same collection that the user is starting with, or items found in a general web search. Implementation, however, suggested the problem that a simple text description of a garment, when fed to a search engine, often retrieves marginally relevant material due to somewhat irrelevant words that wind up in the costume description. For example, the name of a previous owner, although useful in some contexts, is not likely to retrieve documents about costumes in the Web as a whole. One strategy for dealing with this is to use a more systematic vocabulary, in this case the Getty vocabulary subset dealing with costume. The description of an object can be placed in the Getty structure using simple word matching, and then the Getty category name can be used for the larger search.

As an example, the next 3 figures deal with a display of materials that are related to the item the user is considering. Figure 3 below shows the results of taking one item and searching for it in the local catalogue, showing the other related items. Figure 4 is similar but the next row of matches is not from this collection but from Google image search. In Figure 5, a still more complicated process, the item description is looked up in the Getty hierarchy and then the definition from that hierarchy is searched on the web. This example works particularly well because 'wedding dress' is almost a perfect category; it doesn't work nearly so well for cases where words like dress or skirt suggest a large number of possible categories. For this reason, the software supplements the retrieval with other Getty categories so that somebody might reasonably try to search themselves on more appropriate ones, shown at the bottom.

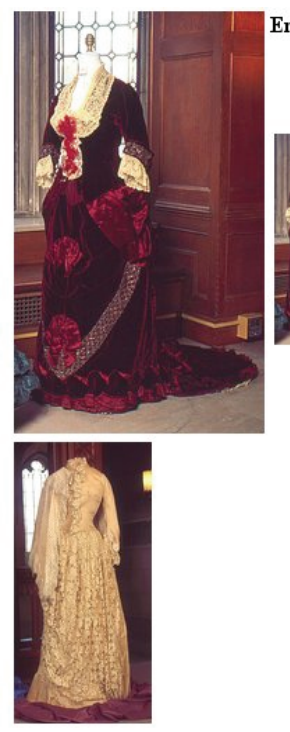

Ensemble of off-white silk satin, taffeta, and lace, with basque bodice, bustled skirt, and short fringed cape, worn by Sallie Tucker Blake at her graduation from Vassar in 1879
Ensemble (dinner dress) of burgundy velvet and satin with beadwork and lace, with basque bodice and bustled skirt
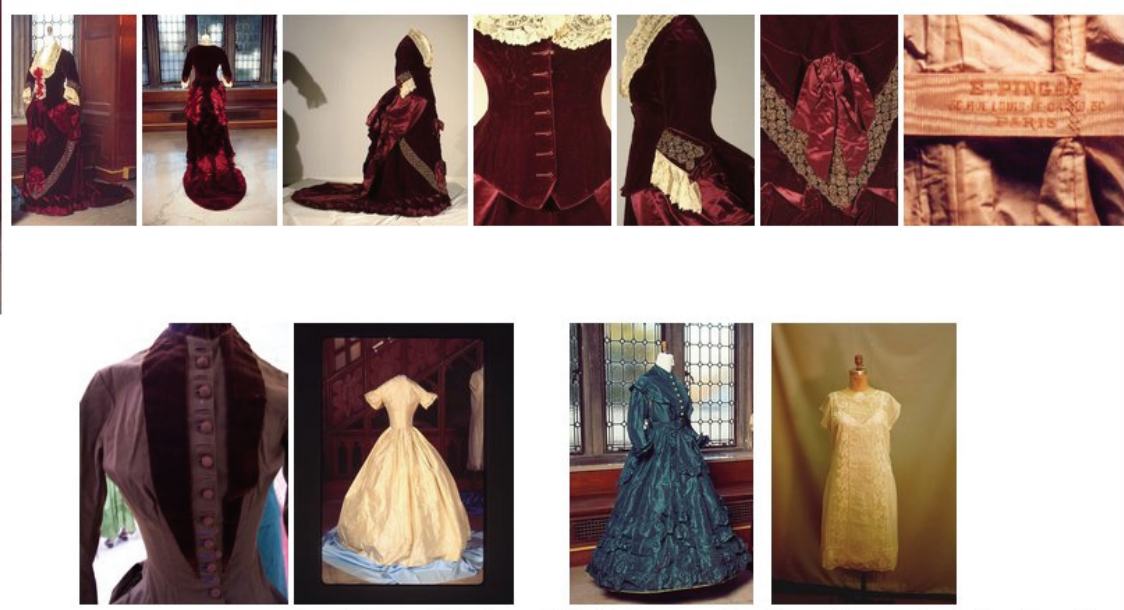

Ivory taffeta wedding dress with Ensemble (day dress) of fitted bodice, short ruffled brown wool and velvet, sleeves, and full pleated
with basque bodice and worn by Margaret A. bustled skirt
Teal taffeta ensemble ruffles on the Daisy Chain at Vassar

shoulders, sleeve hems, Commencement by Kathryn Keeler Sherrill VC â€ 27
Heminway for her wedding on June 21, 1854

Figure 3: Related images within collection only 

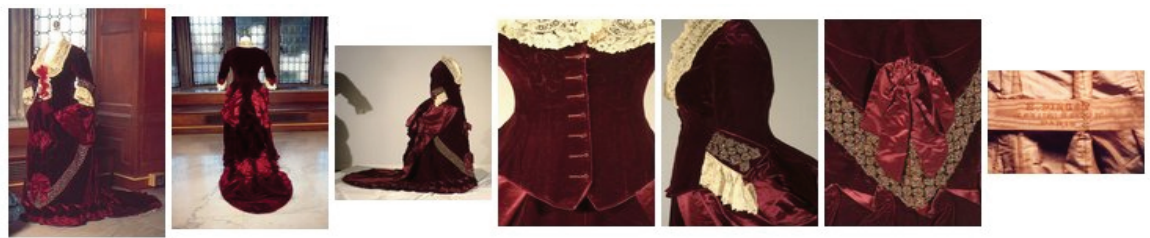

Ensemble (dinner dress) of burgundy velvet and satin with beadwork and lace, with basque bodice and bustled skirt
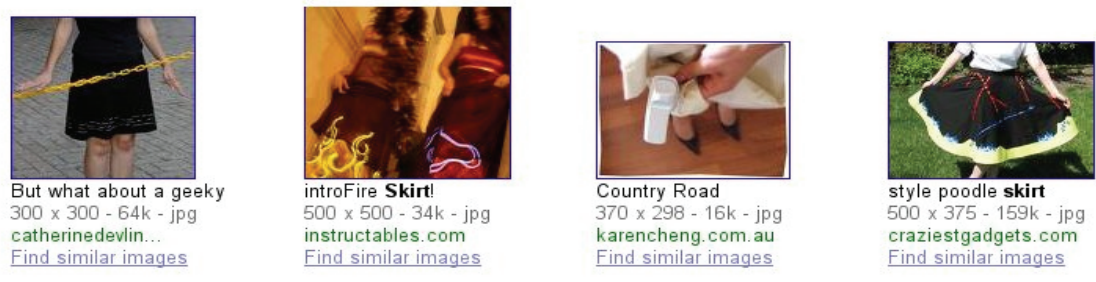

Figure 4: Related images found by web search on image description

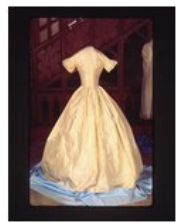

Ivory taffeta wedding dress with fitted bodice, short ruffled sleeves, and full pleated skirt, worn by Margaret A. Heminway for her wedding on June 21, 1854

wedding dresses (subcategory of costume by function) [Getty: 300255177]

wedding dresses: Dresses worn by brides during the wedding ceremony, in many styles and colors, often having a train. weddinggowns, wedding, dresses, bridal, gowns, dress, gown
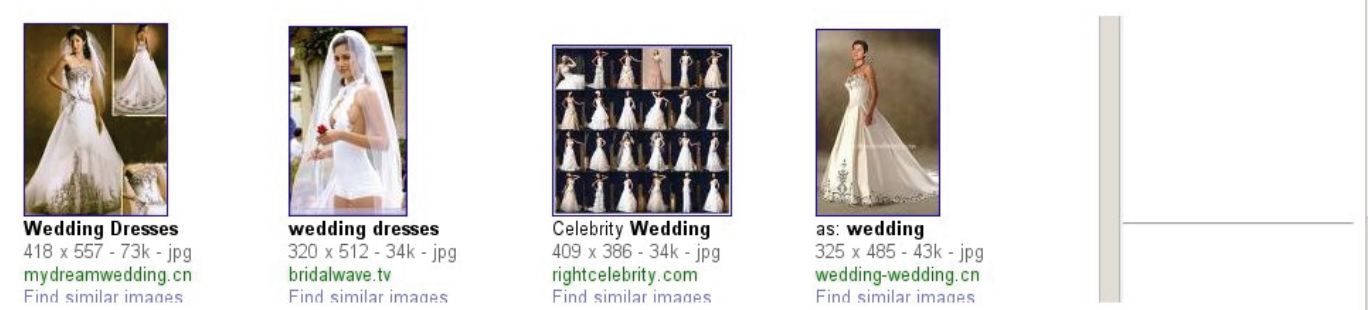

riding skirts: Long, wraparound skirts worn by women for sidesaddle horseback riding. riding-skirts, riding, skirts, skirt

hula skirts (subcategory of costume components) [Getty: 300216041]

hula skirts: Skirts made of long stems of grass bound to a waistband, traditionally worn by Hawaiian hula dancers. Also, imitations of such skirts. skirts, skirt, grass, hula

hanging sleeves (subcategory of costume components) [Getty: 300253738]

hanging sleeves: Long loose sleeves with a slit through which the arm can emerge. Also, long false decorative sleeves attached to the back of the armhole. Worn by men, women, or children especially from the 15 th through the 18 th century. hanging, sleeves, sleeve

skirts (garments) (subcategory of costume components) [Getty: 300209932]

Figure 5: Related images found by web search on text in Getty thesaurus 
What kind of context is most likely to be valuable to a user? This is likely to depend both on the statistics of a particular collection and on the kinds of items being sought. If the user is interested in some rare property, the broadest possible search will be needed, meaning the entire web. More usually, however, the problem will be selection of a few relevant items rather than counting millions of hits on 'costume'. The results of image search on specific categories, however, do often have some extraneous results, and of course the mapping from the description of one item to a formal category name may give inaccurate or misleading results. It might be more useful to allow the user to combine several items when going to a broader context, although that makes the interface more complex.

Screen space is always in short supply, and the correct balance between displaying the current item, the images of items in context, and text descriptions of still other items, again depends on circumstances. We assume in general that users probably want to see more pictures; the specialists who don't need the pictures aren't the ones who are likely to be using interfaces whose purpose is to broaden rather than narrow the materials in a display.

\section{CREATING A 3D REPRESENTATION}

Presentation of a garment as a single static photograph is often inadequate. Aside from the detail pictures of specific parts of a garment, it is valuable to have some kind of representation that shows the costume in space. As Martin (2003) writes, 'Increasing the quality of the data increases its relevance to more users,' justifying her use of high quality rotatable images for a costume collection. Although one can image a full 3D representation, for the purposes of costumes a slightly more restricted view, such as an inverse panoramic view, is good enough (nobody is accustomed to looking at costumes other than right side up). This is created by putting the costume on a mannequin, placing the mannequin on a turntable, and rotating it in front of a camera. A resulting 15 or 24 pictures can then be merged into a single rotatable view, which for obvious reasons can't be printed in a paper document. The representation can easily be either Quicktime VR (QTVR) or Flash (see yofla.com for example software). An example can be seen at http://comminfo.rutgers.edu/ lesk/vassar92.mov.

What is unusual about doing this with costumes? Key differences have to do with preparation and lighting.

a) Since historic costumes are too fragile to be worn by people, they have to be placed on mannequins. Mannequins take longer to dress than people do, since they can't help out in the process. It is important to smooth out all creases, since they won't shake out as somebody moves. Depending on the colour of the garment, the colour of the mannequin and a cloth cover for it will have to be chosen to avoid distractions in the final photographs.

b) To avoid shadows that make part of the costume hard to see, lighting has to be carefully assembled so that all parts of the material are adequately lit. Depending on how sleeves are placed, this can be a somewhat prolonged process and must be done carefully. In addition to the problem of shadowing, the lighting needed to see details of the fabric itself as well as its overall shape may have to be adjusted.

Resolution also needs to be quite high, since users may wish to study the details of fabric, decoration, or design. Despite the increased resolution of digital cameras it is still valuable to take close-up pictures of specific parts of a garment, and under some circumstances lighting needs to be adapted when this is done.

Experience has been that a single large garment still requires perhaps 2 hours, combining dressing the mannequin, setting up the lighting, and taking the photographs. Although some improvements might be forthcoming by using a surplus of lighting equipment the fragility of much historic costume material is always going to make the handling process slow, and keep us from doing these digitisations really rapidly. The implication is that for many years we will probably have costume collections where only a few items have been digitised in panoramic mode, and selectivity by the curators is going to be important.

It is possible to imagine much more elaborate representations. Martin and Ko describe a method for creating virtual people and dressing them in specific costumes (Martin \& Ko, 2009). However, the extra effort in such procedures would mean that fewer items could be included, and acceptability of any system depends on having the items the users care about available in the database.

\section{REFERENCES}

Calhoun, K. (2006) The Changing Nature of the Catalog and its Integration with Other Discovery Tools, Report to the Library of Congress. http://www.loc.gov/catdir/calhoun-report-final.pdf 
Fellbaum, C. (1998) Wordnet: An Electronic Lexical Database. MIT Press, Cambridge.

Fry, E. and Riley, J. (2006) Tagging and UserContributed Metadata.

www.dlib.indiana.edu/ jen/rile/presentations/.../user ContributedMetadata.ppt (April 2010).

Furnas, G. (1986) Generalized fisheye views. In ACM SIGCHI Bulletin, vol. 17, no. 4, pp. 16-23.

Geller, V and M. E. Lesk, M. (1983) User Interfaces to Information Systems: Choices vs. Commands. In Proc. 6th Int. ACM SIGIR Conference, pp. 130135, Bethesda, Md. June 1983.

Gilliland-Swetland, A. (1998) Introduction to Metadata: Pathways to Digital Information, Getty Publications 1998. See page 1.

Martin (2003) User centric design and implementation of a digital historic costume collection. In Proceedings of the American Society for Information Science and Technology, Volume
40, Issue 1, Date: October 2003. pp. 280-290.

Martin, K. and Ko, H. (2009) Virtual historic costume across cultures and disciplines. In 15th International Conference on Virtual Systems and Multimedia, VSMM 09, Vienna, 2009, pp. 104-108.

Stoica, E., Hearst, M. and Richardson, M. (2007) Automating Creation of Hierarchical Faceted Metadata Structures. In Proc. NAACL HLT 2007, Rochester, April 2007. pp. 244-251.

VRA (2010) VRA Core. Visual Resources Assocation.

http://www.vraweb.org/projects/vracore4/ (April 2010).

Wolfe, R. (2010) The value of metadata in the Google era.

http://libraries.mit.edu/metadata/presentations/valu emetadatarev.ppt (April 2010)

Yee, K., Swearingen, K., Li, K. and Hearst, M. (2003) Faceted metadata for image search and browsing. In Proc. SIGCHI conference on human factors in computing systems, 2003, pp. 401-408. 